

\title{
Nouvelles observations sur les fissures radiales du Douglas
}

Hubert Polge

\section{To cite this version:}

Hubert Polge. Nouvelles observations sur les fissures radiales du Douglas. Revue forestière française, 1984, 36 (6), pp.453-458. 10.4267/2042/21757 . hal-03423570

\section{HAL Id: hal-03423570 \\ https://hal.science/hal-03423570}

Submitted on 10 Nov 2021

HAL is a multi-disciplinary open access archive for the deposit and dissemination of scientific research documents, whether they are published or not. The documents may come from teaching and research institutions in France or abroad, or from public or private research centers.
L'archive ouverte pluridisciplinaire HAL, est destinée au dépôt et à la diffusion de documents scientifiques de niveau recherche, publiés ou non, émanant des établissements d'enseignement et de recherche français ou étrangers, des laboratoires publics ou privés. 


\section{biologie et forêt}

\section{NOUVELLES OBSERVATIONS SUR LES FISSURES RADIALES DU DOUGLAS}

\section{H. POLGE}

L'apparition de fissures radiales dans le bois de Douglas à l'état frais a fait l'objet d'une première série d'observations dans un placeau expérimental de l'Arboretum d'Amance (Meurthe-et-Moselle), âgé de 20 ans et planté à un espacement de $1,8 \times 1,8 \mathrm{~m}$; le matériel utilisè était constituè de carottes de sondage de $5 \mathrm{~mm}$ de diamètre, et les principaux points relevès ont èté les suivants (Polge, 1982):

- les fentes ne dépassent généralement pas les limites d'un même accroissement annuel, et sont le plus souvent localisées dans le bois initial ;

- on n'en rencontre pas dans les cernes de l'aubier à humiditè normale, mais dans les zones anormalement sèches de celui-ci (il y a lieu de rappeler que la comparaison de radiographies a l'état frais, puis à l'état $\sec$ d'un même échantillon a permis de montrer que, contrairement à une opinion couramment admise, l'aubier ne présente pas un taux d'humidité uniforme, mais comprend souvent des parties à peu près sèches, parfaitement délimitées et concentrées en général dans le bois initial);

- l'effet de la provenance sur la fréquence des fissures n'est pas net, mais en revanche celui de l'année est très marqué ;

- il existe une liaison très significative, nègative entre la densitè minimale annuelle et le risque de fissuration (en d'autres termes, la probabilité de trouver des fentes dans un accroissement annuel donné est d'autant plus grande que la densité de son bois initial est plus faible) ;

- il n'a pas été possible d'ètablir de liens de cause à effet entre facteurs du climat et fissures radiales, mais l'hypothèse d'une influence de la sècheresse est la plus plausible. 


\section{H. POLGE}

De nouveaux cas de fissures viennent d'être signalés dans des Douglas de 29 ans, plantés à $5 \times 5 \mathrm{~m}$ et fortement élagués (propriété G. Gautier à Chahains, Orne), et la Station de Recherches sur la Qualité des Bois du Centre national de Recherches forestières a eu la possibilité d'examiner quelques-uns des échantillons concernès. II sera fait ètat ci-dessous du résultat de cette étude et des premières conclusions que l'on peut en tirer.

\section{MATÉRIEL ET MÉTHODES}

Quatre planches présentant des fentes et provenant d'arbres différents ont èté réceptionnées, mais l'une d'entre elles, dont le taux d'humidité ètait manifestement au-dessous du point de saturation des fibres, n'a pas été prise en compte, car ses fissures pouvaient être consécutives au retrait du bois et ne pas préexister dans l'arbre sur pied, donc ressortir à un phénomène différent de celui que l'on souhaitait étudier. Dans chacune des trois autres ont été débitées, à l'aide de scies-fraises jumelées, des plaquettes transversales de $1,8 \mathrm{~mm}$ d'épaisseur, à une équidistance théorique de $4 \mathrm{~cm}$, mais pouvant atteindre jusqu'à $15 \mathrm{~cm}$ dans le cas de nœuds ou de distorsions $\mathrm{du}$ fil du bois. Les 30 barrettes ainsi obtenues ont été radiographiées, et les fentes qu'elles comportaient ont été repérées par rapport aux limites des planches, mais aussi par rapport à l'année de production des cernes concernès. Sur les images radiographiques obtenues ont été mesurés au microdensitomètre la largeur des accroissements annuels, leur densite moyenne, minimale et maximale, ainsi que l'écart-type des densités qui caractérise l'hétérogénéité du bois

Pour servir de base de comparaison, les mêmes données ont été relevées sur carottes de sondage dans un certain nombre de Douglas des dispositifs expérimentaux de la Station de Recherches sur la Qualité des Bois d'âge voisin de la parcelle en cause et soumis à des taux d'élagage variables, à savoir :

- 5 arbres au Mont-Pinçon (Calvados) ; taux d'élagage de 32,5 à $45 \%$; densité du peuplement environ 1000 tiges/ha ; âge : 36 ans,

- 10 arbres à Saint-Christophe-de-Chaulieu (Orne); taux d'élagage de 26 à $50 \%$; densité de 400 tiges/ha ; âge : 29 ans,

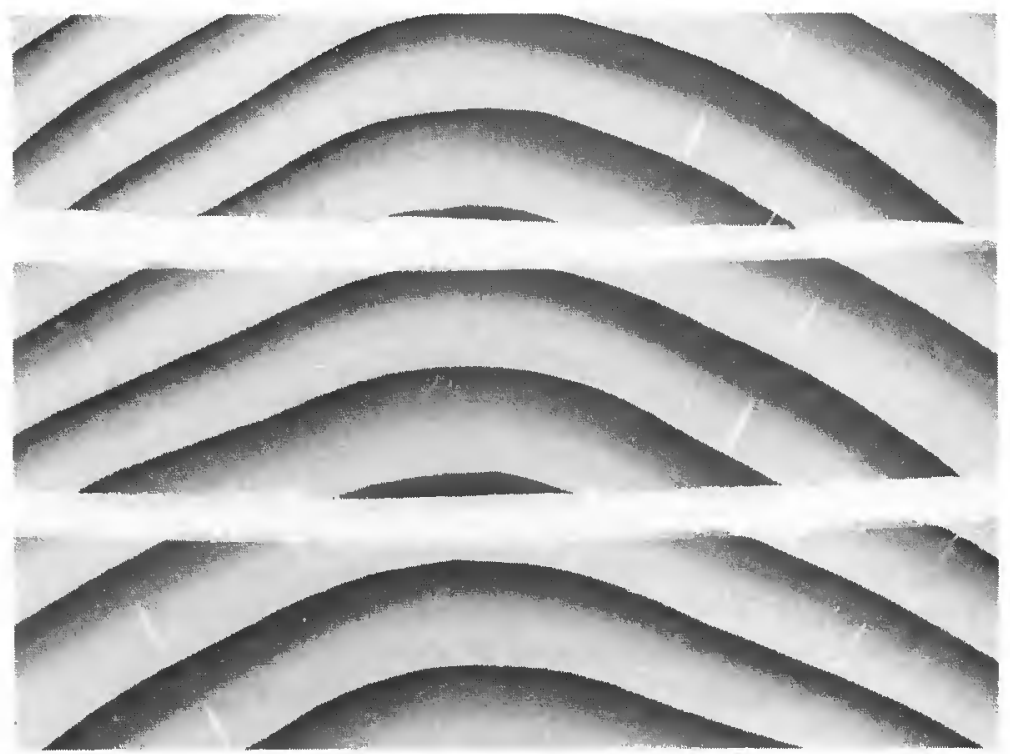

- 3 arbres à Combesalat (Hérault); taux d'élagage de 38 à $41 \%$; densité de l'ordre de 1200 tiges/ha ; âge : 30 ans,

- 5 arbres du sylvetum de Grandsagnes (Hérault) ; taux d'élagage de 0 à $40 \%$; densité voisine de 1600 tiges/ha; âge : 32 ans.

Pour s'affranchir de l'influence connue de l'année sur les caractéristiques densitométriques, celles-ci ont ètè mesurèes sur la tranche de 6 ans de 1969 à 1974 , qui a paru correspondre à celle

Figure 1 : FISSURES RADIALES DANS LE BOIS DE DOUGLAS (3 carottes de sondage). 
des cernes représentés dans les trois planches d'expérience, en se basant sur la position de la limife du bois de cœur coloré, et en estimant le nombre d'annèes d'aubier à 10, c'est-à-dire à celui trouvé à Saint-Christophe-de-Chaulieu, dans des conditions de compétition et d'élagage tout à fait comparables.

\section{RÉSULTATS}

\section{Orientation des fissures}

Comme dans l'expérience d'Amance, elles ont toutes une direction strictement radiale (figure 1), contrairement à l'opinion du scieur qui a débité les bois suivant laquelle elles seraient "plutôt tangentielles ". L'erreur doit sans doute être attribuée au fait que lorsqu'on s'éloigne d'une part de la vraie dosse, d'autre part de la partie centrale des planches, des fentes radiales deviennent de plus en plus inclinées par rapport au plan de sciage, ce qui tend à faire apparaître leur ouverture plus large, surtout après qu'un début de sèchage soit intervenu; par ailleurs, des arrachements de bois peuvent se produire du côté où la fissure fait un angle aigu avec la surface, exagérant ainsi le phénomène.

\section{Dimensions des fissures}

Comme à Amance également, les fentes ne dépassent qu'exceptionnellement les limites de cernes ( 3 cas seulement sur 47 étudiés), et sont, en général, limitées au seul bois initial, d'où, compte tenu de la vigueur des arbres en cause, une dimension radiale de $5 \mathrm{~mm}$ en moyenne.

Dans le sens du fil du bois, le suivi des fentes d'une plaquette à l'autre a montré que leur longueur est très variable, et peut aller de $4 \mathrm{~cm}$ à $50 \mathrm{~cm}$, avec un maximum de fréquence aux environs de $12 \mathrm{~cm}$.

\section{Relation avec les propriétés physiques du bois}

Aucune liaison n'a pu être mise en évidence entre l'existence des fentes et la largeur des accroissements, les densités moyennes ou maximales annuelles, ou encore l'écart-type des densités.

En revanche, une influence possible des densités minimales annuelles se dégage à nouveau, comme à Amance. Le critère retenu est le rapport du nombre de plaquettes où une fente apparaît dans un accroissement annuel donné à celui des plaquettes dans lesquelles ce même cerne est présent; il sera désigné ci-dessous sous le nom de "fréquence de fissures"; c'est une caractéristique qui peut paraître arbitraire, mais qui rend compte de l'importance volumétrique des fentes, puisque le nombre de plaquettes concernées est lié à leur longueur.

Pour l'ensemble des 21 accroissements repérés sur les trois planches étudiées, on ne peut parler que d'une tendance à une aggravation du risque de fissuration lorsque la densité minimale diminue: le coefficient de corrélation trouvé entre celle-ci et la fréquence des fissures n'est que de $-0,39$, signifiant cependant qu'il n'y a que $10 \%$ de chances pour que la liaison observée soit l'effet du hasard. Cependant, si l'on ne considère que la seule planche $n^{\circ} 1$ dans laquelle, à un niveau ou à un autre, 8 cernes se trouvent représentés, la corrélation devient significative: $r=-0,73^{*}$ (figure 2). II est difficile de savoir pourquoi l'influence de la densité minimale ne se manifeste pas avec la même intensitè dans les différentes planches; sans doute ne faut-il pas exclure tout risque d'erreur dans l'identification d'un même accroissement annuel d'un niveau à l'autre en raison de la médiocre rectitude du fil du bois, mais il est sûr que d'autres paramètres interviennent, et notamment la teneur en eau qui influence le module d'élasticité, donc l'aptitude du mafériau à supporter une contrainte mécanique donnée sans se rompre. 


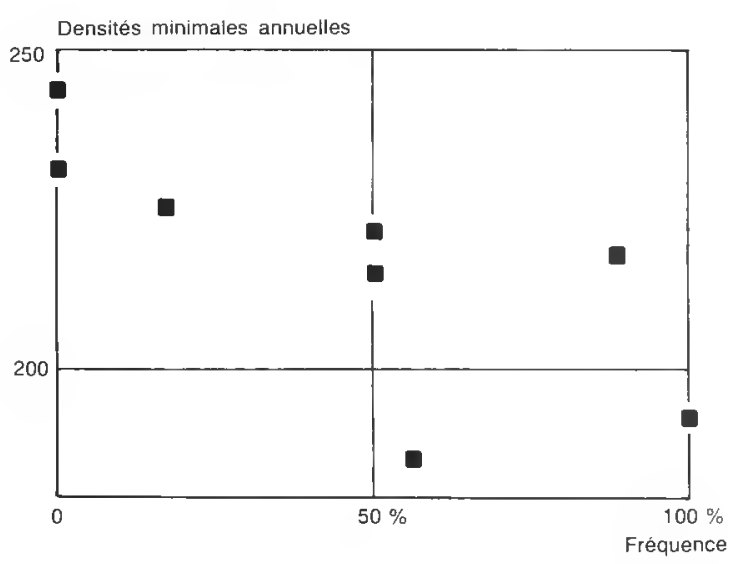

Figure 2 : FRÉQUENCE D'APPARITION DES FISSURES.

Figure 3 : DIAGRAMME DE FRÉQUENCE DES DENSITÉS MINIMALES ANNUELLES.

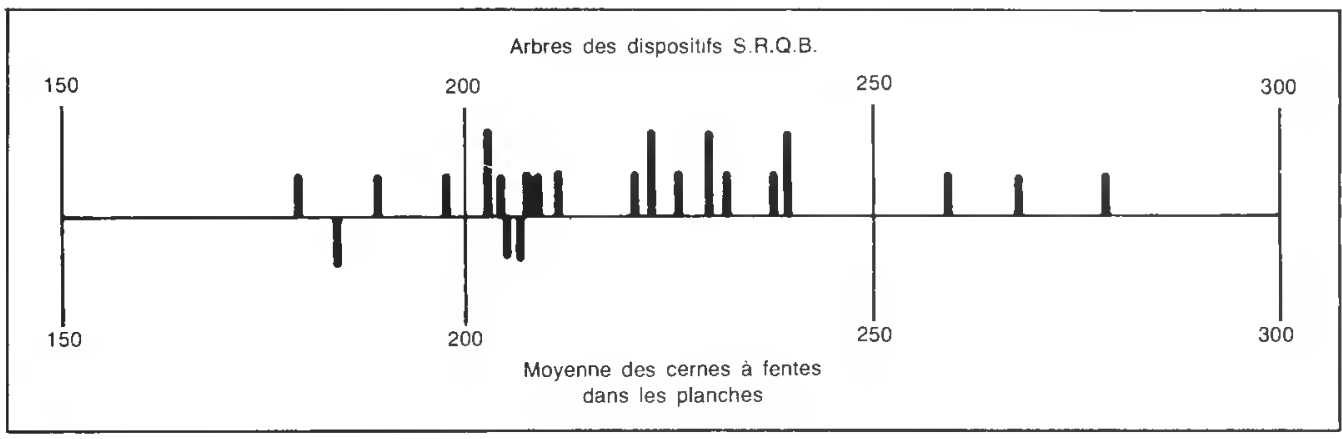

\section{Comparaison avec des Douglas de dispositifs expérimentaux de la Station de Recherches sur la Qualité des Bois}

Sur la figure 3 sont indiquées les densitès minimales moyennes des cernes à fissures des trois planches étudiées par rapport à celles des 22 Douglas sondés dans les dispositifs de la Station; il convient certes d'être conscient des limites de cette comparaison car les arbres en cause ont poussé dans des conditions de compétition, d'èlagage et de milieu différentes. Cependant, une tendance assez nette se dégage en faveur d'une densité minimale relativement faible dans les cernes des planches à fissures. Par ailleurs, même si la probabilitè de rencontrer des fissures radiales dans le petit cylindre que représente une carotte de sondage de $5 \mathrm{~mm}$ augmente avec le nombre et la longueur de celles-ci, il faut bien reconnaître que, sauf densité de fentes très èlevée, il existe un risque sérieux pour que ces prèlèvements passent entre les différentes fissurations; on doit néanmoins signaler que, sur les 22 carottes recueillies, une seule comportait une fissure, et il se trouve que c'est précisément celle dont la densité minimale est la plus faible $\left(179 \mathrm{~g} / \mathrm{dm}^{3}\right)$.

Si l'on ajoute cette carotte à fente radiale aux cernes à fissures des trois planches, on trouve une densité minimale moyenne $\left(194 \mathrm{~g} / \mathrm{dm}^{3}\right)$ qui est significativement inférieure au seuil de $2 \%$ $(t=2,6)$ à celle des 21 autres carottes (densité minimale moyenne de $\left.225 \mathrm{~g} / \mathrm{dm}^{3}\right)$.

Enfin, la densitè minimale moyenne des trois arbres ayant donné des planches à fissures (199 $\left.\mathrm{g} / \mathrm{dm}^{3}\right)$ apparaît encore comme particulièrement faible même si on la compare à la moyenne des seuls arbres du dispositif de Saint-Christophe-de-Chaulieu $\left(217,5 \mathrm{~g} / \mathrm{dm}^{3}\right)$, et ce, bien qu'il s'agisse de peuplements relativement voisins soumis à des conditions très comparables de milieu, 
Figure 4 : LIAISONS ENTRE DENSITÉS MINIMALES ANNUELLES ET TAUX D'ÉLAGAGE.

de compétition et d'élagage. L'ensemble de ces éléments amène à envisager une influence probable du génotype pour expliquer la densité minimale très faible des arbres de la forêt de Chahains.

L'étude des 22 arbres des dispositifs de la Station de Recherches sur la Qualité des Bois permet de faire une observation intéressante quant à l'influence de l'élagage sur la densité minimale du bois: il existe en effet (figure 4) une liaison très significative $\left(r=0,56^{\circ}\right)$ entre celle-ci et le taux d'élagage défini comme le rapport de la longueur de la partie élaguée à la hauteur totale de l'arbre (quand le taux a été modifié pendant la période 1969-1974, un taux pondéré a été calculé sur la base des nombres d'années écoulées avant et après le réélagage). Cette corrélation montre qu'il est sans doute possible d'augmenter la densité minimale, donc de réduire les risques de fissuration, en pratiquant des élagages sévères.

Sans accorder une importance exagérée à cet exemple, on peut signaler que deux des arbres du Sylvetum de Grandsagnes avaient été appariés lors de l'implantation du dispositif, ètant très proches l'un de l'autre et de plus aussi comparables que possible au point de vue dendrométrique ; l'un a été conservé comme témoin avec toutes ses branches, cependant que l'autre ètait élagué au taux moyen de $40,5 \%$; il se trouve que le premier est, des 22 arbres échantillonnés, celui qui a la plus faible densité minimale annuelle $\left(179 \mathrm{~g} / \mathrm{dm}^{3}\right)$, alors que le second a, au contraire, la plus forte $\left(278 \mathrm{~g} / \mathrm{dm}^{3}\right)$.

\section{COMMENTAIRES ET CONCLUSION}

Les conséquences néfastes des fentes radiales du Douglas ne doivent pas être sous-estimées: non seulement elles risquent d'entraîner des pertes de résistance mécanique en cisaillement comme face à tous les autres types de sollicitation, mais encore, elles peuvent présenter un caractère rédhibitoire pour certains emplois prometteurs, s'agissant d'arbres ayant bénéficié d'élagages artificiels de branches vivantes: menuiserie fine ou ébénisterie (à cause du médiocre état de surface qui peut en résulter), déroulage (du fait qu'elles constituent une amorce possible de rupture des placages).

II ne faudrait pas condamner pour autant le Douglas, ni même sa culture à large espacement, car ce défaut n'est apparu jusqu'ici que de façon tout à fait exceptionnelle, et ne semble affecter en outre que la période juvénile de la vie de l'arbre oủ le bois produit est, en tout état de cause, assez peu apte à fournir les emplois technologiques les plus valorisants.

Même si l'on peut déplorer la faiblesse de l'èchantillonnage, ou suspecter d'autres causes de fragilité (module d'élasticité, interaction avec les zones de bois final limitrophes...), il paraît acquis que la densité minimale annuelle joue un rôle important dans l'expression du phénomène, et ceci 


\section{H. POLGE}

ne doit pas surprendre, car il est sûr que, d'un point de vue mécanique, la résistance d'un ensemble à une contrainte donnée est conditionnée par celle de son point le plus faible, ce qui est bien le cas pour la densité minimale de ce matériau composite naturel que constitue le bois.

II est d'autant plus intéressant d'essayer d'agir sur cette caractéristique de structure des accroissements annuels que d'autres expériences ont montré (Keller et Perrin, 1980 ; Thibaut, 1983 ; Nepveu et Tran $\mathrm{Ngoc}, 1984$ ) que la qualité des placages déroulés (ètat de surface, uniformité d'épaisseur) ou des sciages rabotés (état de surface) était d'autant meilleure que la densité minimale annuelle était plus élevée.

Pour augmenter celle-ci, deux cas doivent être envisagès:

- S'agissant des plantations futures, la meilleure solution semble devoir être recherchée dans la voie génétique: un travail récent (Vonnet, 1983) a en effet montré qu'il existait des différences de densité minimale annuelle significatives au seuil de $1 \%$ entre provenances et au seuil de $5 \%$ entre familles ; en outre, compte tenu de l'hèritabilité trouvée $(0,29)$, et avec un taux de sélection de $10 \%$, il apparaît possible de gagner $12 \mathrm{~g} / \mathrm{dm}^{3}$ sur la densité minimale, avec un effet favorable sur les densités maximale et moyenne, sans incidence notable sur l'hétérogénéité, en ne perdant que $3 \%$ et $2,5 \%$ respectivement sur les croissances en hauteur et en diamètre, et en n'avançant la date de débourrement que de deux jours.

- Lorsque l'on a affaire à un jeune peuplement déjà existant, surtout s'il a été planté à faible densité, la pratique d'élagages sévères est la seule possibilité qui reste au sylviculteur pour augmenter la densité minimale du bois produit, mais elle $a$, on l'a vu, toutes chances d'être efficace; il reste que dans le cas de très larges espacements $(5 \times 5 \mathrm{~m}$ et au-delà), même un taux d'ablation des branches vivantes de $50 \%$ peut ne pas être suffisant; il faudrait alors ne pas hésiter à le dépasser pendant la courte période de la vie de l'arbre durant laquelle la croissance risque d'être excessive, donc la densité du bois trop faible; le sacrifice de production consenti en abaissant ainsi la largeur des cernes de 10 à $8 \mathrm{~mm}$ par exemple pendant une douzaine ou une quinzaine d'années devrait être considérè comme un faible prix à payer pour se prémunir contre une dégradation trop grave de la qualité du bois.

Avec la collaboration technique de Pierre GELHAYE et de André PERRIN
H. POLGE

Station de Recherches sur la Qualite des Bois CENTRE NATIONAL DE RECHERCHES FORESTIËRES (I.N.R.A.) CHAMPENOUX 54280 SEICHAMPS

\section{BIBLIOGRAPHIE}

KELLER (R.), PERRIN (J.-R.). - Relations entre les résultats de l'analyse densitomettrique du bois de quelques résineux et la qualité des placages quilis sont susceptibles de produire. - Document á distribution limitée $n^{\circ}$ 1980/4, Station de Recherches sur la Qualite des Bois, C.N.R.F., Champenoux, 1980.

NEPVEU (G.), TRAN NGOC (T.). - Relations entre les composantes densitomètriques et l'état de surface de planches rabotées chez Pseudotsuga menziesii. Annales des Sciences forestières, vol. $41 . n^{\circ} 2.1984$. pp. $171-174$.

POLGE (H.). - Quelques observations sur des fissures radiales dans le bois de Douglas. - Annales des Sciences forestières, vol. 39, $n^{\circ} 4,1982$, pp. 399-406.

THIBAUT (B.). - Étude de l'influence des propriètes mècaniques et structurelles du matériau dans le processus de coupe du bois de déroulage. - Document Laboratoire de Physique des Métaux, Université de Montpellier, 1983.

VONNET (G.). - Contribution à l'ètude de la variabilité génétique de la densitè et de l'hétérogénéitè du bois de Douglas (Pseudotsuga menziesii Mirb.). - D.E.A. de Biologie Végétale, option Sciences forestières, Universite de Nancy, octobre 1983. 